\title{
Surface Modification Effects of Carbon Black on Reinforced Natural Rubber
}

\author{
Hajime Serizawa, Takehusa NaKamura, Masayoshi ITO, \\ Koji TANAKA, and Akimasa NOMURA* \\ Department of Chemistry, Faculty of Science, \\ Science University of Tokyo, \\ Kagurazaka, Shinjuku-ku, Tokyo 162, Japan \\ * Research \& Development Center, Nippon Zeon Co., Ltd., \\ Yako, Kawasaki-ku, Kawasaki, Kanagawa 210, Japan
}

(Received January 13, 1983)

\begin{abstract}
KEY WORDS Natural Rubber / Carbon Black / Pulsed NMR / Spin-Spin Relaxation Time / Bound Rubber / Benzoyl Peroxide / Saponification /
\end{abstract}

It has been reported that the bound rubber in carbon black-rubber systems consists of two components having different segmental mobility, i.e., loosely and tightly bound rubbers, ${ }^{1,2}$ and that the structures of these components are greatly affected by the conditions for preparing the composite ${ }^{3}$ and the nature of carbon black. ${ }^{4}$ We found ${ }^{5}$ that when oxidized carbon black is incorporated into natural rubber, the segmental mobility of both loosely and tightly bound rubbers is enhanced with increasing concentration of acid groups on the carbon black, and simultaneously the loosely bound rubber content is increased. This was explained as due to the weakening of interactions between oxidized carbon black and rubber molecules, probably caused by a decrease in hydrogen atoms $\left(H_{\mathrm{B}}\right)$ bonded to the carbon atoms at the edge of graphitic crystallites in the carbon black. It has been reported that the oxidation of carbon black induces a direct exchange of $H_{\mathrm{B}}$ for the acid group. ${ }^{6}$ However, functional groups such as aldehydes, alcohols, and ketones on the surface of carbon black ${ }^{7}$ may also be transformed into acid groups by oxidation. Therefore, an increase in acid group concentration $\left(C_{\mathrm{f}}\right)$ by oxidation may not directly correspond to a decrease in $H_{\mathrm{B}}$ content. Ohkita et al..$^{8-10}$ reported that $H_{\mathrm{B}}$ is exchanged for the benzoyloxy group by the reaction of carbon black with benzoyl peroxide (BPO treatment), and also that the benzoyloxy group is transformed into the hydroxyl group by saponification
(BPO-SP treatment). Thus, the increase in benzoyloxy or hydroxyl groups introduced into the carbon black may correspond to the decrease in $H_{\mathrm{B}}$. By incorporation of BPO or BPO-SP treated carbon black into natural rubber, it should be possible to obtain direct information on the previously suggested relation ${ }^{5}$ between the change in $H_{\mathrm{B}}$ content and the bound rubber structure. In this study, pulsed NMR measurements were carried out on bound rubber prepared from the composite of BPO or BPO-SP treated carbon black and natural rubber, and the effects of surface modification of carbon black on the bound rubber structure were examined.

\section{EXPERIMENTAL}

\section{Samples}

The carbon black and rubber used in this study were Diablack A (Mitsubishi Chemicals Co. Ltd.) and pale crepe (natural rubber), respectively. The oily matter on Diablack A was extracted with benzene for at least $50 \mathrm{~h}$ in a Soxhlet extractor, and the purified carbon black was dried at $100^{\circ} \mathrm{C}$ in vacuo (untreated carbon black). Benzoyl peroxide (BPO) treated and saponified (BPO-SP) carbon blacks were obtained using the procedure reported by Ohkita et al. ${ }^{10}$ The untreated carbon black was allowed to react with benzoyl peroxide in carbon tetrachloride for $50 \mathrm{~h}$ at $45^{\circ} \mathrm{C}$ with occasional shak- 
ing. By so doing, $H_{\mathrm{B}}$ was exchanged for benzoyloxy

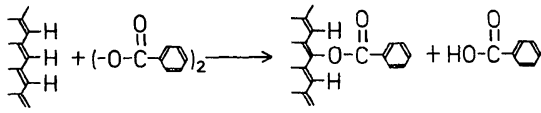

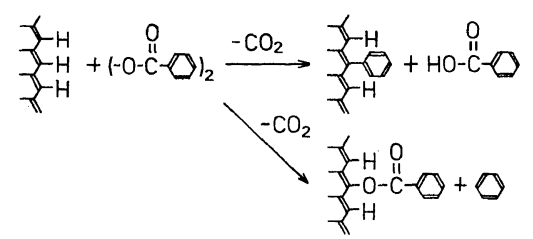

groups (eq 1) ${ }^{8,9}$ In this treatment, the secondary reaction shown in eq 2 was considered minor, since it is expected to occur only at high temperature. ${ }^{8}$ To remove unreacted BPO and benzoic acid produced during the reaction, the reaction mixture was washed several times with carbon tetrachloride at room temperature and then with benzene for at least $100 \mathrm{~h}$ in a Soxhlet extractor. The saponification of BPO treated carbon black was carried out in the following way. The BPO treated carbon black was added to a small excess of aqueous sodium hydroxide (about $0.1 \mathrm{~mol} \mathrm{dm}^{-3}$ ) and the mixture was held at $95^{\circ} \mathrm{C}$ for $1 \mathrm{~h}$ with slow stirring. The reaction mixture was then acidified with hydrochloric acid. With this treatment, the benzoyloxy groups on the BPO treated carbon black are transformed into

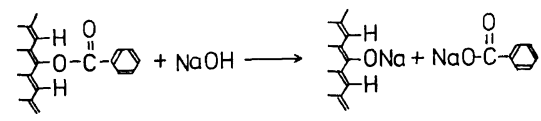

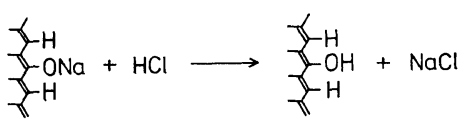

phenolic hydroxyl groups (eq 3). ${ }^{9,10}$ The treated carbon black was washed carefully with hot distilled water and then dried at $100^{\circ} \mathrm{C}$ in vacuo. The $C_{\mathrm{f}}$ of the BPO-SP treated carbon black was determined by the base adsorption method ${ }^{5}$ in aqueous sodium hydroxide. The $C_{\mathrm{f}}$ of the BPO treated carbon black could not be determined by the neturalization technique, since benzoyloxy groups are saponified by sodium hydroxide. However, the number of benzoyloxy groups and the $C_{\mathrm{f}}$ are equal to that of the phenolic hydroxyl groups on the BPOSP treated carbon black and the $C_{\mathrm{f}}$ of untreated carbon black, respectively. The oxidized carbon black, prepared by a method described elsewhere was used for comparison. Carbon gel samples were prepared from the composites of treated carbon blacks and natural rubber by the method described in our previous paper. ${ }^{5}$

\section{Measurement}

Pulsed NMR measurements were performed with a JEOL pulsed NMR spectrometer (JSE-5B), operating at $60 \mathrm{MHz}$. The proton spin-spin relaxation time $\left(T_{2}\right)$ was determined by the solid echo sequence with a pulse width of $2 \mu$ s and a pulse interval of $9 \mu$ s. Solid echo signals were accumulated using a time-averaging computer. The $T_{2}$ and fraction of each component were determined by the method described in the previous paper. ${ }^{3}$

\section{RESULTS AND DISCUSSION}

Table I shows the surface area, total acid concentration $\left(C_{\mathrm{f}}\right)$ and decrement of $H_{\mathrm{B}}\left(\Delta H_{\mathrm{B}}\right)$ of carbon black. The surface area was determined by the B.E.T. method using nitrogen adsorption. Virtually, no change in surface area was caused by oxidation, BPO and BPO-SP treatments. Thus, the observed changes in segmental mobility and fraction of bound rubber, induced by incorporation of the treated carbon black into natural rubber, are directly related to the surface modification of carbon black as discussed below. By BPO-SP treatment, $C_{\mathrm{f}}$ increased from 0.14 to $0.29 \mathrm{meq} \mathrm{g}^{-1}$. This increment in $C_{\mathrm{f}}$ corresponds to the $\Delta H_{\mathrm{B}}$ of BPO-SP and BPO treated carbon black as described in the previous section. The oxidation of carbon black also induces an increase in $C_{\mathrm{f}}$. In addition to the exchange of $H_{\mathrm{B}}$ for the acid group, ${ }^{6}$ functional groups such as aldehydes, alcohols, and ketones on the carbon black may be transformed into acid groups by oxidation. ${ }^{7}$ Therefore, the value of $\Delta H_{\mathrm{B}}$ for oxidized carbon black may be less than $0.13 \mathrm{meq}^{-1}$.

Table I. Characteristics of carbon black

\begin{tabular}{lccc}
\hline Carbon black & $\begin{array}{c}\text { Surface } \\
\text { area } / \mathrm{m}^{2} \mathrm{~g}^{-1} \\
\pm 5\end{array}$ & $\begin{array}{c}C_{\mathrm{f}} / \mathrm{meq}^{-1} \\
\pm 0.02\end{array}$ & $\Delta H_{\mathrm{B}} / \mathrm{meq} \mathrm{g}^{-1}$ \\
\hline Untreated & 133 & 0.14 & 0 \\
Oxidized & 121 & 0.27 & $<0.13$ \\
BPO treated & 133 & $(0.14)$ & 0.15 \\
BPO-SP treated & 132 & 0.29 & 0.15 \\
\hline
\end{tabular}


Figure 1 shows the temperature dependence of $T_{2}$ for carbon gel samples (CG-U and CG-S). Here, CG-U and CG-S were prepared from the composites of natural rubber and untreated carbon black and the BPO-SP treated carbon black, respectively. Above $-40^{\circ} \mathrm{C}$, both samples exhibit two $T_{2}$ 's, indicating the existence of long $T_{2}\left(T_{2 \mathrm{~L}}\right)$ and short $T_{2}\left(T_{2 \mathrm{C}}\right)$ component, i.e., loosely and tightly bound rubbers. In all the samples, a sharp increase in $T_{2 \mathrm{~L}}$ was found in the range from -40 to $20^{\circ} \mathrm{C}$, where a small increase in $T_{2 \mathrm{C}}$ was also found in the CG-S sample. The changes in $T_{2 \mathrm{~L}}$ and $T_{2 \mathrm{C}}$ with temperature are probably due to the high frequency $(\sim 10 \mathrm{kHz})$ manifestation of the glass-rubber transition of the samples. Above the transition temperature, the temperature dependence of $T_{2 \mathrm{~L}}$ and $T_{2 \mathrm{C}}$ is weak and the $T_{2 \mathrm{~L}}$ and $T_{2 \mathrm{C}}$ component fractions $\left(f_{\mathrm{L}}\right.$ and $f_{\mathrm{C}}$ ) in the total bound rubber remain almost constant (data not shown). All the samples showed a similar temperature dependence of $T_{2 \mathrm{C}}, T_{2 \mathrm{~L}}, f_{\mathrm{C}}$,

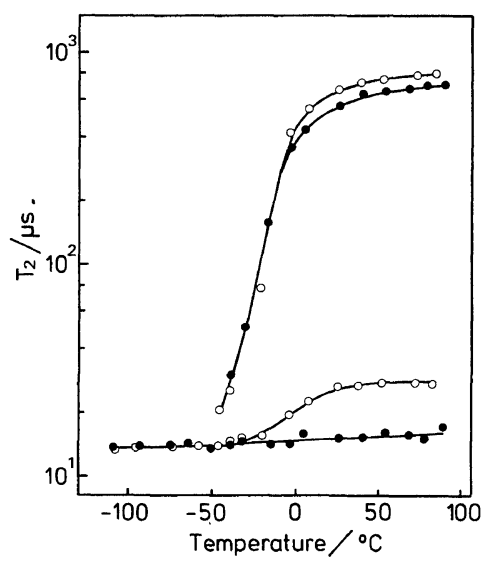

Figure 1. Temperature dependence of $T_{2}$ for carbon gel samples (CG-U and CG-S):, , CG-U prepared from the composite of untreated carbon black with natural rubber; $\mathrm{O}, \mathrm{CG}-\mathrm{S}$ prepared from the composite of BPOSP treated carbon black with natural rubber. and $f_{\mathrm{L}}$. Therefore, the values of the $T_{2}$ 's and fractions $\left(f_{\mathrm{C}}\right.$ and $\left.f_{\mathrm{L}}\right)$ of both bound rubbers at $40^{\circ} \mathrm{C}$ (Table II) were used for the evaluation of the bound rubber structure. In this table, the fraction of the total bound rubber $\left(F_{\mathrm{BR}}\right)$, and the fractions of the $T_{2 \mathrm{C}}\left(F_{\mathrm{C}}\right)$ and $T_{2 \mathrm{~L}}\left(F_{\mathrm{L}}\right)$ components in the filled rubber were determined by the method described in our previous paper. ${ }^{5}$ We found ${ }^{5}$ that by incorporation of oxidized carbon black with different acid group concentrations into natural rubber, $F_{\mathrm{L}}, F_{\mathrm{BR}}$, and the segmental mobility in the $T_{2 \mathrm{C}}$ and $T_{2 \mathrm{~L}}$ phases increase with increasing $C_{\mathrm{f}}$. The present study indicated that the oxidation effects of carbon black on the bound rubber content $\left(F_{\mathrm{BR}}, F_{\mathrm{C}}\right.$, and $\left.F_{\mathrm{L}}\right)$ and the segmental mobility in the $T_{2 \mathrm{C}}$ and $T_{2 \mathrm{~L}}$ phases are not appreciable, probably due to the low value of $C_{\mathrm{f}} \cdot{ }^{5}$ As seen in Tables I and II, CG-S shows a $F_{\mathrm{BR}}$ value higher than $\mathrm{CG}-\mathrm{O}$, despite the fact that these samples have similar $C_{\mathrm{f}}$ values. This means that the increase in $F_{\mathrm{BR}}$ cannot be related directly to the increase in $C_{\mathrm{f}}$. On the other hand, the samples with high values of $\Delta H_{\mathrm{B}}$ had large $F_{\mathrm{BR}}$ values. Thus, the changes in $F_{\mathrm{BR}}$ are more sensitive to $\Delta H_{\mathrm{B}}$ than $C_{\mathrm{f}}$. Table II shows that the samples containing larger amounts of tightly and loosely bound rubbers have longer $T_{2 \mathrm{~L}}$ and $T_{2 \mathrm{C}}$. The increase in $T_{2 \mathrm{~L}}$ and $T_{2 \mathrm{C}}$ implies an increase in segmental mobility in the $T_{2 \mathrm{~L}}$ and $T_{2 \mathrm{C}}$ phases and suggests a decrease in the interaction between carbon black and rubber molecules. Papirer et al. ${ }^{11}$ suggest that $H_{\mathrm{B}}$ has something to do with the chemisorption of unsaturated rubber on carbon black. If so, the chemical adsorptive activity of carbon black should decrease with increasing $\Delta H_{\mathrm{B}}$. It is generally accepted that the bound rubber phase surrounding the carbon black consists mainly of long and short loops. ${ }^{4}$ Thus, a reduction in the number of active points on the carbon black may cause a decrease in the number of loops. If the number of loops diminishes with increasing $\Delta H_{\mathrm{B}}$ with no change in the length of

Table II. Properties of bound rubber

\begin{tabular}{llccccc}
\hline $\begin{array}{c}\text { Carbon gel } \\
\text { sample }\end{array}$ & Carbon black & $\begin{array}{c}F_{\mathrm{BR}} \\
\pm 0.010\end{array}$ & $\begin{array}{c}T_{2 \mathrm{C}} / \mu \mathrm{s} \\
\pm 1\end{array}$ & $\begin{array}{c}T_{2 \mathrm{~L}} / \mu \mathrm{s} \\
\pm 10\end{array}$ & $\begin{array}{c}F_{\mathrm{C}} \\
\pm 0.004\end{array}$ & $\begin{array}{c}F_{\mathrm{L}} \\
\pm 0.008\end{array}$ \\
\hline CG-U & Untreated & 0.366 & 16 & 664 & 0.041 & 0.325 \\
CG-O & Oxidized & 0.379 & 21 & 681 & 0.040 & 0.339 \\
CG-B & BPO treated & 0.450 & 25 & 690 & 0.052 & 0.398 \\
CG-S & BPO-SP treated & 0.430 & 27 & 730 & 0.050 & 0.380 \\
\hline
\end{tabular}




\section{H. Serizawa et al.}

loops, the total bound rubber content $\left(F_{\mathrm{BR}}\right)$ should decrease with increasing $\Delta H_{\mathrm{B}}$, in contrast to the present results. The increase in segmental mobility and bound rubber content in the $T_{2 \mathrm{~L}}$ and $T_{2 \mathrm{C}}$ phases, induced by the decrease in the number of active points on the carbon black, may be explained as follows. As a result of the decrease in the number of anchored points of rubber molecules, the length of the loops on the carbon black increases, leading to an increase in $T_{2 \mathrm{~L}}$ and $T_{2 \mathrm{C}}$, and simultaneously in both $F_{\mathrm{C}}$ and $F_{\mathrm{L}}$. The reduction in loops contributed to the bound rubber content is compensated and/or overcome by the increase in loop length. The previous study revealed no clear change in $F_{\mathrm{C}}$ with $C_{\mathrm{f}}$. However, CG-B an CG-S showed clear changes in $F_{\mathrm{C}}$ with $\Delta H_{\mathrm{B}}$ (Tables I and II), suggesting that the chemical adsorptive activity of carbon black can be decreased more effectively by the BPO and/or BPOSP treatment than by oxidation of the carbon black. The $T_{2 \mathrm{~L}}$ and $T_{2 \mathrm{C}}$ of CG-B were slightly shorter than those of CG-S, despite the very small difference in $F_{\mathrm{BR}}$ of these samples. At present, the reason is unknown, but it is true that constraint on the segmental motion of the bound rubber in CG-B is stronger than that in CG-S. The benzoyloxy group is fairly bulkier than that of other groups, and may hinder the segmental motion of the bound rubber phase.

In summary, the changes in segmental mobility and fraction of bound rubber are larger in the BPO or BPO-SP treated carbon black-natural rubber system, i.e., these changes are more sensitive to the change in the number of $H_{\mathrm{B}}$ than $C_{\mathrm{f}}$. The decrease in the number of $H_{\mathrm{B}}$ induces an increase in segmental mobility and fractions of both bound rubber phases. The results can be explained as resulting from an increase in loop length on the carbon black owing to the decrease in the interaction between treated carbon black and natural rubber. It may be concluded that BPO or BPO-SP treated carbon black rather than oxidized carbon black is suitable for controlling the content and structure of bound rubber in a carbon black-natural rubber composite.

\section{REFERENCES}

1. S. Kaufman, W. P. Slichter, and D. D. Davis, J. Polym. Sci., A-2, 9, 829 (1971).

2. T. Nishi, J. Polym. Sci., Polym. Phys. Ed., 12, 685 (1974).

3. H. Serizawa, M. Ito, T. Kanamoto, K. Tanaka, and A. Nomura, Polym. J., 14, 149 (1982).

4. J. O'Brien, E. Cashell, G. E. Wardell, and V. J. McBrierty, Macromolecules, 9, 653 (1976).

5. H. Serizawa, T. Nakamura, M. Ito, K. Tanaka, and A. Nomura, Polym. J., 15, 201 (1983).

6. D. Rivin, Rubber Chem. Technol., 44, 307 (1971).

7. M. L. Studebaker, Rubber Age, 77, 69 (1955).

8. K. Ohkita, H. Kasahara, N. Ishizuki, and Y. Itagaki, Nippon Gomu Kyokaishi, 36, 361 (1963).

9. K. Ohkita and N. Tubokawa, Carbon, 10, 631 (1972).

10. K. Ohkita, N. Tubokawa, K. Sekine, and T. Yamada, Nippon Gomu Kyokaishi, 47, 40 (1974).

11. E. Papirer, A. Voet, and P. H. Given, Rubber Chem. Technol., 42, 1200 (1969). 